\title{
TUMOR SÓLIDO-CÍSTICO PSEUDOPAPILAR DO PÂNCREAS MULTICÊNTRICO SUBMETIDO À GASTRODUODENOPANCREATECTOMIA TOTAL: RELATO DE CASO E REVISÃO DA LITERATURA
}

\section{Pseudopapillary Solid-cystic multicentric pancreatic tumor submitted to total gastroduodenopancreatectomy: Case report and literature review}

\author{
Alexandre Cruz HENRIQUES, Sergio Renato Pais COSTA, Mauricio Campanelli COSTAS, \\ Jaques WAISBERG, Manlio Basílio SPERANZINI
}

ABCDDV/561

Henriques AC, Costa SRP, Costas MC, Waisberg J, Speranzini MB. Tumor sólido-cístico pseudopapilar do pâncreas multicêntrico submetido à gastroduodenopancreatectomia total: relato de caso e revisão da literatura. ABCD Arq Bras Cir Dig 2007;20(3):212-5.

RESUMO - Racional - O tumor sólido-cístico pseudopapilar do pâncreas é neoplasia rara. Acomete mais comumente indivíduos jovens do sexo feminino e tem sido considerada neoplasia de baixo grau de malignidade com comportamento biológico indolente. O seu tratamento tem sido a ressecção cirúrgica. Pode comprometer tanto a cabeça quanto o corpo ou mesmo a cauda do pâncreas. Contudo, a presença de duas lesões simultâneas, uma na porção cefálica e outra na transição do corpo para a cauda do pâncreas (multicentricidade) é situação muito rara. Relato de caso - Tumor sólido-cístico pseudopapilar do pâncreas multicêntrico (com duas lesões distintas na cabeça e no corpo-cauda) em homem de 36 anos de idade submetido à gastroduodenopancreatectomia total e esplenectomia. $\mathrm{O}$ exame histológico revelou a presença de dois tumores distintos em cabeça $\mathrm{e}$ corpo-cauda do pâncreas, ambos de mesma etiologia (sólido-cístico pseudopapilar) e cuja confirmação foi realizada por avaliação imunoístoquimica. O paciente teve boa evolução pós-operatória. Cinco meses após o tratamento cirúrgico não apresenta sinais de recidiva. Conclusão - Esses tumores apresentam bom prognóstico, com curabilidade e todos os esforços devem ser tentados para a sua ressecção mesmo que para isto seja necessária pancreatectomia total.

DESCRITORES - Neoplasias pancreáticas. Pancreatectomia.

\section{INTRODUÇÃO}

Tumor Sólido-Cístico Pseudopapilar do Pâncreas (TSCPP) é tumor raro. Foi descrito pela primeira vez por Frantz em 1959 que reportou quatro casos e até então era desconhecida como neoplasia anteriormente considerado como tumor de ilhotas pancreáticas não-funcionantes. Contudo, descreveu essa neoplasia como uma nova entidade e a nomeou de "tumor papilífero do pâncreas".

Diversas denominações têm sido dadas a esse tumor ao longo de sua história: neoplasia epitelial papilífera, neoplasia cística papilífera, neoplasia papilífera sólido-cística, tumor sólido-cístico acinar, neoplasia sólida papilífera, carcinoma cístico papilífero, tumor papilífero sólido-cístico e tumor sólido pseudopapilífero ${ }^{2}$. Contudo em 1996, em consenso da World Health Organization foi finalmente designado de tumor sólido-cístico pseudopapilar do pâncreas ${ }^{9,11}$.

Esse tumor corresponde a apenas 5\% dos tumores pancreáticos císticos e um a 2\% de todos os tumores exócrinos desse órgão ${ }^{2,5,8,16}$. Em passado recente, tem ocorrido aumento da sua freqüência, em parte pelo maior conhecimento de

Trabalho realizado no Serviço de Cirurgia Geral do Hospital de Ensino da Faculdade de Medicina do ABC, Santo André, SP, Brasil.

Endereço para correspondência: Sergio Renato Pais, e-mail:sergiorenatopais@terra.com.br sua existência e da maior identificação por métodos de imunoistoquímica ${ }^{16}$.

Poucas séries de casos dessa neoplasia têm sido descritas no Brasil, porém nenhum caso de TSCPP multicêntrico havia sido descrito anteriormente ${ }^{3,4,10}$. Os autores descrevem um caso de paciente com duas lesões simultâneas, na cabeça e no corpo-cauda do pâncreas (multicêntrico) que foi submetido a gastroduodenopancreatectomia total com esplenectomia com boa evolução pós-operatória.

\section{RELATO DO CASO}

Homem de 36 anos de idade, negro, deu entrada no Serviço de Cirurgia Geral do Hospital de Ensino da Faculdade de Medicina do $\mathrm{ABC}$ com sintoma de dor epigástrica associada ao aparecimento de massa abdominal. Simultaneamente apresentava emagrecimento de $5 \mathrm{~kg}$ em seis meses. Ele não apresentava doenças associadas.

Foram realizadas ultra-sonografia de abdome e endoscopia digestiva alta que não apresentavam alterações, e tomografia computadorizada de abdome (Figura 1) que mostrou presença de duas volumosas lesões nodulares sólidas, de contorno regulares, com realce heterogêneo do meio de contraste, medindo respectivamente $6 \times 5 \times 4 \mathrm{~cm}$ e 10,8 X 5,1 X 6,1 cm, localizadas em região retro-gástrica, 
a menor na região proximal do pâncreas (cabeça) e a maior na sua região distal (corpo-cauda). Foram ainda dosados os marcadores CEA, CA19-9 e CA125 que se apresentavam dentro da normalidade.

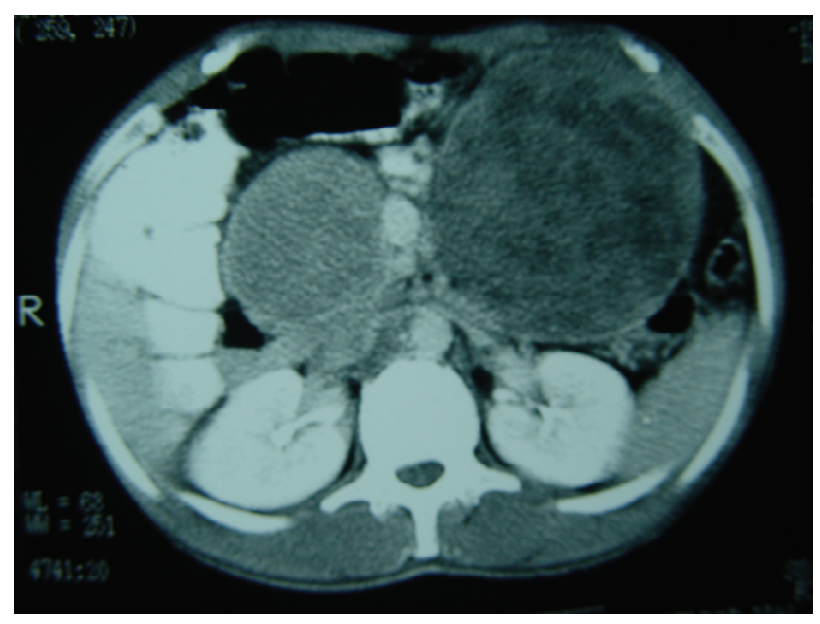

FIGURA 1 - Tomografia de abdome mostrando duas lesões sincrônicas, heterogêneas em cabeça e corpo-cauda pancreáticos.

Subsequentemente, ressonância nuclear magnética de abdome foi realizada. Foram observadas, duas volumosas lesões expansivas em pâncreas (cabeça e corpo-cauda) que apresentavam coeficiente de atenuação heterogêneo, com o predomínio de hipersinal em T2 e hiposinal em T1 respectivamente. Essas lesões apresentavam ainda realce predominantemente periférico após administração do contraste paramagnético. A lesão na porção distal do pâncreas media cerca de $10 \mathrm{~cm}$ de diâmetro enquanto a localizada na porção cefálica cerca de $5 \mathrm{~cm}$ de diâmetro.

Como havia dúvida na ressecabilidade, o estadiamento foi complementado com ecoendoscopia e arteriografia de vasos mesentéricos. A primeira, revelou a presença de duas lesões discretamente hipoecóicas e homogêneas - uma menor (cerca de $5 \mathrm{~cm}$ de diâmetro) localizada em cabeça pancreática e outra maior na porção distal do órgão -. Ambas apresentavam planos de clivagem bem delimitado, sem dilatação ou acometimento do hepatocolédoco ou ducto pancreático principal. A lesão de localização cefálica deslocava o tronco mesentérico-portal, porém não o invadia. $\mathrm{Na}$ arteriografia não se observou invasão de grandes vasos.

Frente a este quadro o paciente foi levado à laparotomia exploradora com proposta de pancreatectomia total. $\mathrm{O}$ achado intra-operatório revelou pâncreas com dois grandes tumores de aspecto neoplásico, um acometendo a região cefálica e outro a região corpo-caudal. Havia ainda pequena área de tecido pancreático de aspecto normal posicionada entre a cabeça e o corpo. Também foi observada linfoadenopatia locoregional, acometendo tanto o tronco celíaco quanto o mesocólon transverso. A princípio foi realizada pancreatectomia distal com esplenectomia (Figura 2) e linfadenectomia regional, sendo o material enviado para biópsia de congelação. O exame revelou tratar-se tumor sólido- cístico pseudopapilar do pâncreas com processo inflamatório reacional em linfonodos. Frente a esse achado, a operação foi complementada com gastroduodenopancreatectomia cefálica (Figura 3). O paciente evoluiu bem, tendo alta no $14^{\circ}$ dia de pós-operatório.

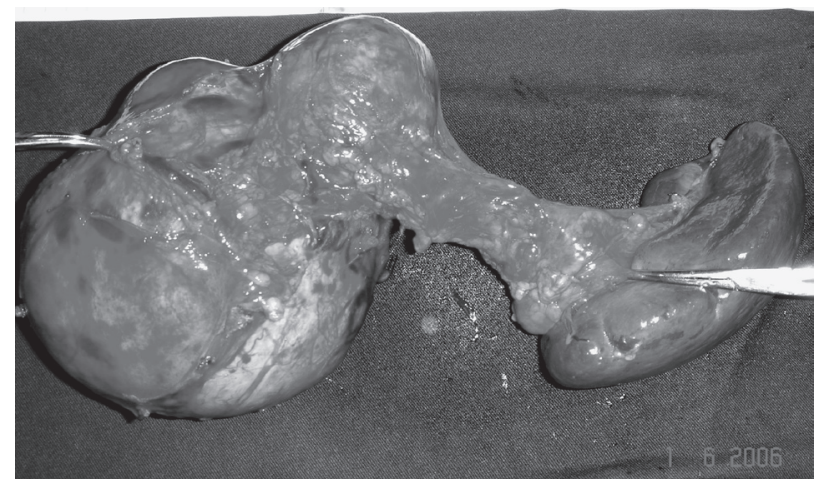

FIGURA 2 - Espécime cirúrgico mostrando grande lesão em corpo-cauda do pâncreas

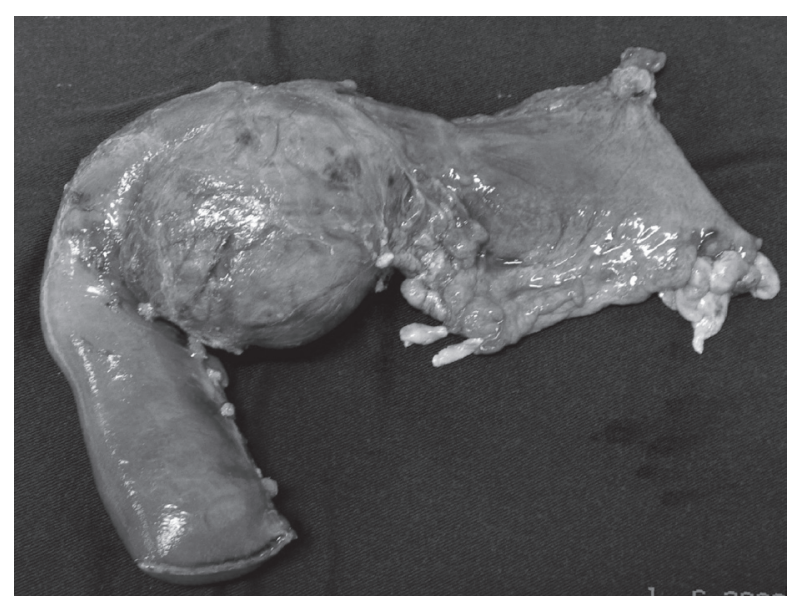

FIGURA 3 - Espécime cirúrgico mostrando também grande lesão tumoral em porção cefálica do pâncreas.

O exame anátomo-patológico revelou a presença de um tumor sólido-cístico pseudopapilar do pâncreas (Tumor de Gruber-Frantz) em ambas as lesões, com focos de hemorragia e necrose. As margens de ressecção estavam livres de neoplasia como também os linfonodos regionais. $\mathrm{O}$ painel imunoistoquímico esta demonstrado na Tabela 1 e foi compatível com tumor de Frantz nas duas lesões.

TABELA 1 - Painel imunoistoquímico

\begin{tabular}{lc}
\hline Marcador imunoistoquímico & Positivo (focal) \\
\hline Cromogranina A & Positivo \\
Vimentina & Positivo \\
Alfa 1-antiquimotripsina & Positivo \\
Alfa 1-antitripsina & Positivo \\
Enolase Neuron-Específica & Positivo (focal) \\
S100 & Positivo (focal) \\
Receptores de progesterona & Positivo \\
\hline
\end{tabular}


No último retorno, cinco meses após a operação, o paciente encontra-se bem e sem queixas. A insuficiência endócrina está sendo compensada com insulina, e a exócrina com reposição via oral de enzimas pancreáticas.

\section{DISCUSSÃO}

TSCPP são neoplasias incomuns e representam somente $2,5 \%$ de todos tumores pancreáticos ${ }^{16}$. Desde que foram descobertos, apenas 718 casos foram relatados até o ano de $2005^{14}$. Recentemente, porém esses números vem se elevando, muito devido ao melhor conhecimento histológico dessa entidade ${ }^{1,13,15}$. O pico de incidência se situa em torno das segunda e terceira décadas de vida. Esses tumores apresentam predileção pelo gênero feminino. Sua origem é motivo de grande controvérsia. Para determinados autores, tem origem em células primordiais multipotentes do pâncreas, enquanto para outros é extra-pancreática. Embora alguns estudos discutam sua origem em detalhe, a linhagem de diferenciação celular ainda permanece incerta ${ }^{5,6,12,14,17,18}$.

Os TSCPP têm sido encontrados tanto na cabeça como no corpo e cauda do pâncreas ${ }^{11}$. Contudo, lesões multicêntricas são muito raras e em revisão de literatura, apenas um outro caso foi previamente encontrado ${ }^{13}$. Como sua freqüência é baixa, seu diagnóstico é muitas vezes negligenciado. Como diagnósticos diferenciais têm sido descritas lesões neoplásicas, tanto metastáticas quanto primárias, cistos linfoepiteliais e pseudocistos secundários à pancreatite ${ }^{3,4,10,12}$.

Esses tumores têm sido considerados como de baixo grau de malignidade. Em geral são encapsulados e raramente invadem estruturas contíguas. Apresentam crescimento lento e raramente se disseminam por via linfática. Metástases hematogênicas são raras, com poucos casos descritos na literatura. No entanto, quando presentes, o fígado tem sido o órgão mais comumente acometido ${ }^{1,6,13,15}$.

Devido a seu lento crescimento, esses tumores podem alcançar grandes dimensões, mormente por ocasião de seu diagnóstico. Os sintomas mais descritos têm sido dor (abdominal ou lombar), dispepsia ou mesmo a presença de massa abdominal ${ }^{1,3,4,5,8,10,13}$. Geralmente, esses tumores tornam-se sintomáticos justamente quando atingem grandes proporções. O diâmetro médio das lesões sintomáticas tem se situado em torno de $6 \mathrm{~cm}$ (mais que $75 \%$ deles são maiores do que $5 \mathrm{~cm}$ ). Em contrapartida, casos assintomáticos representam somente $15,5 \%$ dos casos relatados ${ }^{1,10,12,15}$. $\mathrm{O}$ caso descrito, com dois tumores de grandes dimensões, coincide com esses achados, pois o paciente apresentava tanto dor quanto massa abdominal.

O diagnóstico é feito por exames de imagem. Os incluídos tem sido ultra-sonografia, tomografia computadorizada e menos freqüentemente ressonância nuclear magnética. $\mathrm{O}$ achado mais comumente observado na tomografia computadorizada é massa heterogênea, arredondada e encapsulada (bem definida), com áreas sólidas e císticas. Ocasionalmente, a lesão pode apresentar septações ou calcificações em seu interior. Embora incomum, massas de aparência sólida como observadas no presente caso também podem ser observadas ${ }^{12,13,17}$. Para diagnóstico complementar a ultrasonografia endoscópica pode ser utilizada, principalmente em casos de dúvida diagnóstica ou mesmo para avaliação da ressecabilidade, como foi possível de se observar no presente caso. A biópsia por ecoendoscopia, em algumas situações pode corroborar para seu diagnóstico preciso, mormente para os casos de difícil diagnóstico etiológico ${ }^{13,15}$. Os marcadores tumorais séricos CEA e CA19-9 se apresentam de maneira geral dentro dos valores normais como observado no presente caso, e pouco contribuem para o seu diagnóstico ${ }^{8,9,10,11}$. Em raras ocasiões, mínimas elevações do CA19-9 podem ser relatadas $^{1,15,16}$.

$\mathrm{Na}$ análise macroscópica, esses tumores apresentam áreas sólidas amareladas e císticas, com zonas hemorrágicas e necróticas. A histologia é caracterizada por áreas sólidas que se alternam com áreas pseudopapilíferas com espaços císticos de permeio como observado no presente caso $^{2,16}$. O padrão imunoistoquímico é muito diverso. As células são consistentemente positivas para vimentina, alpha-1-antitripsina e alpha-1- antiquimiotripsina como foi observado no presente caso. Algumas vezes são focalmente positivas para enolase neuron-específica e receptores de progesterona como no caso reportado ${ }^{14,17}$. Mais recentemente, tem sido descrita a positividade para CD10 e CD56 ${ }^{12}$.

O tratamento tem sido a ressecção cirúrgica, pois esses tumores apresentam baixa resposta tanto à radioterapia quanto à quimioterapia ${ }^{1,2,8,14,15}$. Esses tumores apresentam bom prognóstico, com grande curabilidade e todos os esforços devem ser tentados para a sua ressecção. A operação indicada depende da localização do tumor no pâncreas. Lesões no corpo ou na cauda têm sido tratadas de preferência, com pancreatectomia distal e preservação esplênica. Para lesões pequenas localizadas no colo pancreático, a pancreatectomia central pode ser opção ${ }^{7,18}$. Para as lesões na região cefálica, tanto a duodenopancreatectomia com preservação pilórica, quanto a gastroduodenopancreatectomia cefálica têm sido as técnicas de eleição. A linfadenectomia regional é raramente indicada, exceto se linfonodos aumentados ou suspeitos estão presentes ${ }^{1,2,8,14,15,18}$. Mesmo na presença de metástases hepáticas, a ressecção tanto da lesão primária quanto das lesões hepáticas com o intuito curativo deve ser indicada ${ }^{2,6,14,15}$. Como lesões multicêntricas são incomuns, a gastroduodenopancreatectomia total é raramente indicada. Porém, deve ser recomendada com precisão nessa excepcional situação clínica, pois se trata de tumor de bom prognóstico compensando desta maneira a grande morbidade de ambas insuficiências tanto exócrina quanto endócrina ${ }^{13}$.

\section{CONCLUSÃo}

Os tumores sólido-cístico pseudopapilares do pâncreas apresentam bom prognóstico, com curabilidade e todos os esforços devem ser tentados para a sua ressecção mesmo que para isto seja necessária pancreatectomia total. 
Henriques AC, Costa SRP, Costas MC, Waisberg J, Speranzini MB. Pseudopapillary solid-cystic multicentric pancreatic tumor submitted to total gastroduodenopancreatectomy: case report and literature review. ABCD Arq Bras Cir Dig 2007;20(3):212-5.

ABSTRACT - Background - Solid-cystic pseudopapillary pancreatic tumors are rare neoplasms. Female young individuals are usually struck by this condition. This type of tumor has been considered to be a low-grade neoplasia, having an indolent biological behavior. Surgical ressection has been the treatment of choice. This disease can also compromise the head, body as well as the tail of the pancreas. However, the presence of two simultaneous lesions, one in the cephalic portion and the other at the transition between the body and tail of the pancreas (multicentricity) is a very rare situation. Case report - Solid-cystic multicentric pseudopapillary pancreatic tumor (with two distinct lesions, one in the head and the other in the body-tail) in a 36 year old male was submitted to total gastroduodenopancreatectomy and splenectomy. Histological tests revealed the presence of two distinct tumors, one in the head and the other in the body-tail of the pancreas, both of the same etiology (solid-cystic pseudopapillary tumor), receiving confirmation by immunohistochemical evaluation. The patient had a good post-operative development. Five months after surgical treatment, the patient did not show any signs of recidive. Conclusion - This tumors present a good prognosis and cure, so every effort should be taken to resect the tumor, even if it is necessary to perform total pancreatectomy.

HEADINGS - Pancreatic neoplasms. Pancreatectomy.

\section{REFERÊNCIAS}

1. Bahri I, Njim L, Khabir A, Mahmoudi H, Ghorbel A, Zakhama A, Jlidi R. Tumeur papillaire solide et cystique du pancreas. Ann Chir. 2001;126:899902.

2. Casadei R, Santini D, calculli L, Pezzilli R, Zanini N, Minni F. Pancreatic solid-cystic papillary tumor: clinical features, imaging findings and operative management. JOP J Pancreas. 2006;7:137-44.

3. Costa-Neto GD, Amico EC, Costa GI. Tumor sólido-cístico pseudopapilar do pâncreas (tumor de Frantz). Estudo de quatro casos. Arq Gastroenterol. 2004;41:259-62.

4. Cunha JE, Machado MC, Penteado S, Jukemura J, Abdo EE, Bacchella T, Montagnini AL. Tratamento dos tumores císticos do pâncreas. In: Atualização em cirurgia do aparelho digestivo e coloproctologia. São Paulo: Frôntis; 2002. p.187-95.

5. Frantz VK. Tumors of the pancreas. In: Anonymous atlas of tumor pathology. Washington: Armed Forces Institute of Pathology; 1959. p.32-3.

6. Horisawa M, Ninomi N, Sato T, Yokoi S, Oda K, Ichikawa M, Hayakawa S. Frantz's tumor (solid and cystic tumor of the pancreas) With liver metastasis: sucessful treatment and long-term follow-up. J Ped Surg. 1995;30:724-6.

7. Iacono C, Bortolasi L, Serio G. Indications and techinique of central pancreatectomy - early and late results. Langenbecks Arch Surg. 2005;390:266-71.

8. Klimstra DS, Wenig BM, Heffess CS. Solid-pseudopillary tumor of the pancreas: a typically cystic carcinoma of low malignat potential. Semin Diagn Pathol. 2000; 17:66-80

9. Kloppel G, Solcia E, Longnecker DS, Capella C, Sobin LH. Histological typing of tumors of the exocrine pancreas. New York: Springer; 1996.
10. Machado MC, Monteiro da Cunha JE, Bacchella T, Jukemura J, Penteado S, Zerbini MC, et al. Tumor de pâncreas (neoplasia epitelial e cística do pâncreas): estudo de três casos. Rev Hosp Clín Fac Méd São Paulo. 1993;48:29-34.

11. Martin RC, Klimtra DS, Brennan MF, Conlon KC. Solid-pseudopapillary tumor of the pancreas: a surgical enigma? Ann Surg Oncol. 2002;9:35-40.

12. Notohara K, Hamazaki S, Tsukayama C, Nakamoto S, Kawabata K, Mizobuchi K. Solid-pseudopapillary tumor of the pancreas, immunohistochemical localization of neuroendocrine markers and CD10. Am J Surg Pathol. 2000;24:136171.

13. Orlando CA, Bowman RL, Loose JH. Multicentric papillary-cystic neoplasm of the pancreas. Arch Pathol Lab Med. 1991;115:958-60.

14. Papavramidis T, Papavramidis S. Solid pseudopapillary tumors of the pancreas: review of 718 patients reported in english literature. J Am Coll Surg. 2005;6:965-72.

15. Raffel A, Cupisti K, Krausch M, Braunstein S, Trobs B, Goretzki PE, Willnow $\mathrm{U}$. Therapeutic strategy of papillary cystic and solid neoplasm (PCSN): a rare non-endocrine tumor of the pancreas in children. Surg Oncol. 2004;13:1-6.

16. Santero Ramirez MP, Gonzalvo E, Martinez A, Del Rio Marco FJ. Tumor papilar solido quístico de páncreas. Tres formas distintas de presentacion. Rev Esp Enferm Dig. 2004;96:285-6.

17. Santini D, Poli F, Lega S. Solid-papillary tumors of the pancreas: histopathology. JOP J Pancreas (Online). 2006;7:131-6.

18. Spinelli KS, Fromwiller TE, Daniel RA, Kiely JM, Nakeeb A, Komorowiski RA, Wilson SD, Pitt HA. Cystic pancreatic neoplasms. Ann Surg. 2004;239:651-9.

Conflito de interesse: não há

Fonte financiadora: não há

Recebido para publicação em: 04/02/2007

Aceito para publicação em: 16/05/2007 